\title{
In-situ Broadband Cryogenic Calibration for Two-port Superconducting Microwave Resonators
}

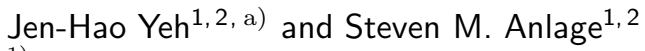 \\ ${ }^{1)}$ Electrical and Computer Engineering Department, University of Maryland, College Park, \\ Maryland 20742-3285 \\ ${ }^{2)}$ CNAM, Physics Department, University of Maryland, College Park, Maryland 20742-4111
}

(Dated: 12 March 2013)

\begin{abstract}
We introduce an improved microwave calibration method for use in a cryogenic environment, based on a traditional three-standard calibration, the Thru-Reflect-Line (TRL) calibration. The modified calibration method takes advantage of additional information from multiple measurements of an ensemble of realizations of a superconducting resonator, as a new pseudo-Open standard, to correct errors in the TRL calibration. We also demonstrate an experimental realization of this in-situ broadband cryogenic calibration system utilizing cryogenic switches. All calibration measurements are done in the same thermal cycle as the measurement of the resonator (requiring only an additional 20 minutes), thus avoiding 4 additional thermal cycles for traditional TRL calibration (which would require an additional 12 days). The experimental measurements on a wave-chaotic microwave billiard verify that the new method significantly improves the measured scattering matrix of a high-quality-factor superconducting resonator.
\end{abstract}

PACS numbers: 84.40.-x, 07.20.Mc, 06.20.fb, 06.30.Ka, 07.57.Pt, 05.45.Mt

\section{INTRODUCTION}

Microwave resonators are widely-utilized devices in many science and engineering fields. In order to achieve very low dissipation and attain extremely high quality factors $Q$, microwave resonators can be made of superconducting materials at temperatures lower than the critical temperature $T_{c}^{1,2}$. One important application of superconducting resonators is in building particle accelerators. They can transfer energy to a charged particle beam with high efficiency because superconducting resonators can store the energy with very low loss and a narrow bandwidth ${ }^{3}$.

Another application of superconducting resonators, and also the motivation of the microwave calibration method in this paper, is to test the predictions of wave chaos theories in a low loss (nearly unitary) regime ${ }^{4}$. Wave chaos, or quantum $\operatorname{chaos}^{5}$, is a field where researchers study the manifestations of chaotic dynamics of classical trajectories on the short-wavelength (quantum or wave) properties of wave systems. Random Matrix Theory (RMT) ${ }^{6}$ has successfully been applied to predict many statistical properties of open wavechaotic systems, such as the scattering matrices $(S)$, the impedance matrices $(Z)$, the conductivities, and the fading amplitudes ${ }^{4}-12$ in complicated wave scattering environments. There has been great success in studying the properties of wave chaotic systems in the context of microwave billiards 5 .

For the scattering matrix $S$ of an open wave-scattering system, the prediction of RMT is for the universal features of the statistical distributions of the elements of $S$

\footnotetext{
a) davidyeh@umd.edu
}

and their correlations, which should only depend on the loss parameter of the system ${ }^{7-10}$. The loss parameter $\alpha$ is defined as the ratio of the $3 \mathrm{~dB}$-frequency bandwidth of the cavity resonances due to distributed losses, to the average spacing between resonant frequencies, and it can be expressed as $\alpha=k^{2} /\left(\triangle k_{n}^{2} Q\right)$, where $k$ is the wave number for the incoming wave, $\triangle k_{n}^{2}$ is the mean spacing of the adjacent eigenvalues of the Helmholtz operator $\left(\nabla^{2}+k^{2}\right)$ of the corresponding closed system, and $Q$ is the loaded quality factor of the cavity ${ }^{8.9}$. Examination of the predictions of RMT in very low loss systems is interesting because extreme limits for the distribution functions and other predictions are encountered ${ }^{7,8,12}$. For example, in a two-port time-reversal invariant system, the distribution of the magnitude of $\left|S_{21}\right|$ can be approximated by a Rayleigh distribution when the system has a moderate or high loss parameter, but the distribution $P\left(\left|S_{21}\right|\right)$ goes to a uniform distribution when the loss parameter goes to zero ${ }^{7,12}$.

For a practical network system, the measured scattering matrix contains both the universal features predicted by RMT and system-specific features which are not included in the prediction of RMT. The system-specific (non-universal) features of the wave scattering properties can be removed by methods such as the random coupling model (RCM) $9,11,13,14$ or the Poisson Kernel ${ }^{15}$. In order to test the statistical predictions, we use a two-port superconducting microwave resonator as the wave-chaotic billiard to create a low-loss experimental system, and we measure the $2 \times 2$ scattering matrix $S$ of the superconducting cavity in a wide frequency band. We then apply the random coupling model to reveal the universal features from the measured scattering matrix to compare with the predictions of RMT.

To measure the scattering matrix $S$ with high accuracy over a broad bandwidth, a broadband calibration 
method of microwave measurement is necessary. The calibration method removes the effect of the transmission lines connecting the vector network analyzer (VNA) at room temperature and the cryogenic sample at low temperature. Because the convenient electronic-calibration kit of a commercial VNA does not function in a cryogenic environment, one must perform a manual calibration by utilizing known standards.

\section{REVIEW OF CRYOGENIC MICROWAVE CALIBRATION}

Microwave calibration is an important process to remove the systematic errors due to the transmission lines and connectors between the network analyzer and the device under test (DUT) as well as other systematic measurement errors. The calibration process utilizes measurements of known standards to move the reference plane of the measurement to the ports of the DUT 16.17 . A commonly-used calibration method for two-port measurement is the Thru-Reflect-Line (TRL) calibration $\frac{18,19}{}$.

The Thru-Reflect-Line calibration uses three standards to calibrate the effect of the two transmission lines connecting the two ports of the DUT to the VNA. For the Thru standard measurement, the two transmission lines are directly connected together; an additional electrically short (on the order of one guided wavelength) transmission line is added between the two transmission lines as the Line standard; two identical reflectors are connected to the ends of the two transmission lines as the Reflect standard $\underline{19}$. The advantages of the TRL calibration are from two facts: (i) the use of redundant calibration standards reduces the uncertainty due to errors, such as connector irreproducibility, cable flexure, test-set drift, and noise, and (ii) the foundation of the calibration standard definitions depend solely on qualitative requirements (uniformity of the lines, identical cross-sections of the lines, and identical reflection coefficients of the Reflect standards) 20 .

For applying TRL calibration in cryogenic systems, one challenge is that measuring multiple standards may involve cooling down and warming up of the system, changing the standard, and repeating the thermal cycle. These thermal cycles can be very time consuming and expensive. In addition, there is enhanced uncertainty of the reproducibility of experimental conditions in different thermal cycles 17.20 . On the other hand, researchers have developed single-thermal-cycle calibration methods which use an on-wafer cryogenic probe station or electromechanical switches. However, the problem of these single-thermal-cycle methods is that the differences between electrical paths in measurements of different standards, which are assumed to be equal, degrade the measurement accuracy and limit the frequency bandwidth ${ }^{17}$.

For one-port systems, a typical calibration method is the Open-Short-Load (OSL) calibration. A pioneering work on broadband cryogenic calibration of a one-port system was done by Booth et al $\stackrel{21}{n}$ who measured the complex reflection coefficient $S_{11}$ of a superconducting thin film. By taking advantage of the superconductive feature, they cooled the sample deep into the superconducting state and used it as a short-circuit standard to correct their OSL calibration of the Corbino reflectometer at room temperature (or temperatures higher than the critical temperature $T_{c}$ ). Other researchers also applied the OSL calibration and the superconductingshort-standard approach in their one-port cryogenic measurement ${ }^{22-24}$. Instead of using the superconductingshort-standard approach, Reuss and Richard ${ }^{25}$ also measured broadband $S_{11}$ of a superconducting thin film, but they applied the 3 -standard (Open, Short, Load) calibration, once at room temperature and once at the superconducting temperature (for a total of 6 calibration measurements). One problem with the OSL calibration is that it is very sensitive to the standards, and one must carefully reproduce all connections, bending and twisting of the transmission lines, and the temperature distribution in the apparatus $21-23,25$. Kitano et al. 26 used the normal-state conductivity of a sample as a Load standard to address this problem.

For two-port systems, researchers have developed cryogenic TRL calibration methods for measurement of the $2 \times 2$ scattering matrix of a DUT. Laskar et al $l^{27}$ utilized the multiline method of the TRL and LRM (Line-ReflectMatch) calibrations introduced by Marks ${ }^{19}$, to their cryogenic on-wafer probe station for noise and scatteringparameter measurements, and they emphasized the importance of providing a stable thermal environment 27 . Booth et al. also used a cryogenic probe station for scattering-parameter measurements of their coplanar waveguide $(\mathrm{CPW})$ structures in high temperature superconductors ${ }^{28,29}$. They applied a set of CPW calibration structures of TRL standards to characterize the errors in the network analyzer/probe station system ${ }^{28}$, or alternatively three other standards: a Thru, a Reflect, and a series resistor ${ }^{30}$. Shemelin et al. used the TRL calibration for waveguides and coaxial cables to measure ferrites at low temperature 31 . In addition to the TRL calibration method, Jun et al. use a different calibration method by introducing a cryogenic dip probe for timedomain measurements of nanodevices ${ }^{32}$.

Another way to achieve single-thermal-cycle TRL calibration is to utilize cryogenic microwave switches 33 . Ranzani et al ${ }^{34}$ use cryogenic switches (coaxial subminiature latching switches) to switch the coaxial cables from the VNA to coaxial cables with different calibration standards, as well as the device under test. The electromechanical switches simply operate by means of brief electrical pulses to latch the switch to different positions, so no electrical signal is applied to the switch in its quiescent state. Due to the convenience of connecting the coaxial transmission lines to the ports of our superconducting cavity, we use cryogenic switches to develop the in-situ calibration system. Similar cryogenic switches have been 
applied for calibrating measurement of Superconducting Quantum Interference Device (SQUID) amplifiers ${ }^{35}$, measuring different superconducting qubit samples ${ }^{36}$, or other experiments involving superconducting quantum computing 37 .

Although the TRL calibration is less sensitive to the properties of the standards than the OSL calibration, the TRL calibration is still limited by errors in its assumptions, such as irreproducibility of the transmission lines in each measurement, differences in the reflection coefficients of the two reflectors, and irreproducibility of the connector interface ${ }^{20,38}$. Researchers have tried different methods to reduce the calibration errors, such as the development of precise dimensional characterization techniques for the transmission lines 39 , the minimization of the possible center-conductor-gap variation $\underline{40}$, and modeling of the electrical properties combined with selfcalibration approaches ${ }^{20.41}$. In our cryogenic measurements, the temperature dependence of the scattering matrices of all transmission lines and imperfect TRL standards become additional sources of errors. These small errors are especially significant in our extremely-low loss system because the calibrated $\left|S_{11}\right|$ and $\left|S_{22}\right|$ are very close to 1 away from the resonance frequencies.

In this paper, we introduce a self-calibration approach by taking advantage of multiple measurements in different realizations of the superconducting resonator. We call this additional information a pseudo-Open standard and utilize it to correct the measured scattering matrices after the TRL calibration. With the improvement provided by the pseudo-Open standard, we also demonstrate an experimental result utilizing this in-situ broadband cryogenic calibration system.

\section{CALIBRATION METHOD}

\section{A. The Superconducting Resonator}

We have carried out experiments by measuring the complex $2 \times 2$ scattering matrix $S$ of a quasi-twodimensional microwave cavity, illustrated in Fig. 1] There are two coupling ports (the red cylinders and dots in Fig. 1) where microwaves are injected through an antenna attached to a single-mode coaxial transmission line of characteristic impedance $Z_{0}=50 \Omega$. Each antenna is inserted into the cavity through a small hole in the lid, similar to previous setups 9.12 . The waves introduced have frequencies from 3 to $18 \mathrm{GHz}$, and they are quasi-two-dimensional due to the thin height of the cavity $(0.8 \mathrm{~cm}$ in the $z$ direction). The antennas are terminated with SMA connectors, one male and the other female, on the surface of the resonator. The shape of the cavity is a "cut-circle" and is a billiard potential that shows classical chaos $12.42-44$.

The superconducting cavity is made of copper with $\mathrm{Pb}$ plated walls and cooled to a temperature $(6.6 \mathrm{~K})$ below the transition temperature of $\mathrm{Pb} \underline{43}-\underline{45}$. Measurements of

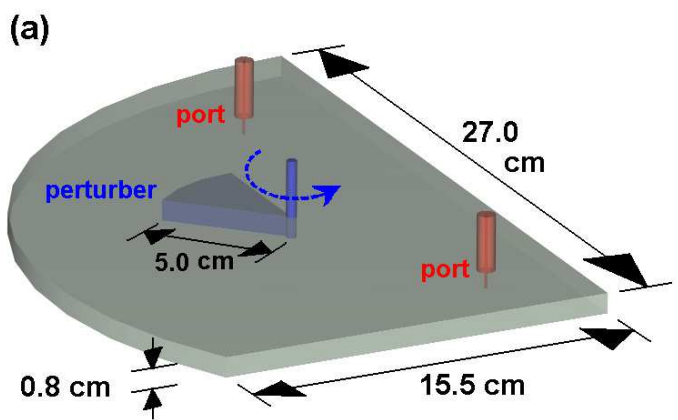

(b)

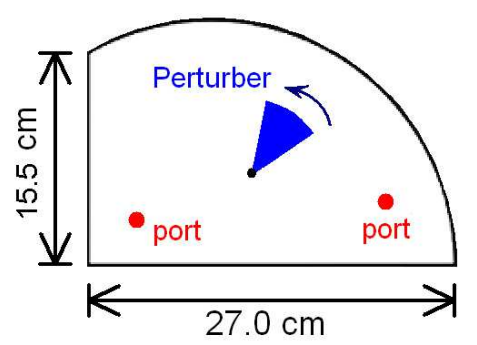

FIG. 1. (a) The quasi-two-dimensional cut-circle microwave cavity in a three-dimensional perspective, showing the cavity dimensions, ports, and the perturber. (b) Projected twodimensional view of the cut-circle billiard.

the transmission spectrum suggest that the quality factor of the resonances is on the order of $Q \approx 10^{5}$. A Teflon wedge (the blue wedge in Fig. 10) can be rotated as a ray-splitting perturber inside the cavity, and we rotate the wedge by $5^{\circ}$ each time to create an ensemble of 72 different realizations. The rotation axis of the perturber is connected with a MDC vacuum rotary feedthrough (BRM-133) at room temperature, so we can control the angular position from outside the cryostat. The original purpose of creating these realizations was to gather statistics to test the statistical predictions of RMT, but it is now a critical procedure for the pseudo-Open standard.

\section{B. The In-situ Broadband Cryogenic Calibration System}

Figure 2 shows the setup of our in-situ broadband cryogenic calibration system. The term "in-situ" means the TRL calibration process can be applied at low temperatures without spending a great deal of time changing standards. Here we utilize two cryogenic 6-position switches (Radiall coaxial subminiature latching switches R591722605) to include one Thru, one Reflect, and two Line standards together with the cavity under measurement. Each switch is connected to different standards, or to the cavity, by RF COAX phase-matched (13 inch long between interfaces, electrical length deviations $<1 \mathrm{ps}$ ) SMA coaxial cables (S086MMHF-013-1). These cables have male SMA connectors in the both ends. Therefore, for the Thru standard, we connect a pair of the coaxial cables with a female-female adapter (Mini-Circuits adapter SF-SF50+). In order to make sure the electrical paths 
are as identical as possible, the same type of industriallyassembled cables, and adapters, have been used for the other 4 pairs of transmission lines. In future experiments, we plan to replace half of the phase-matched coaxial cables by cables terminated with one male connector and one female connector. In this way we will not need the female-female adapters and can reduce the number of connectors in each electrical path, so the number of error sources can also be reduced. For the Reflect standard, we use two short circuits, one with a male connector and the other with a female connector (Fairview Microwave models SC2136 and SC2141), to terminate the pair of coaxial cables. For the Line standards, we add two different SMA male-to-female adapters of electrical length 1.94 $\mathrm{cm}$ and $2.56 \mathrm{~cm}$ (Fairview Microwave models SM4971 and SM5291) to connect the two pairs of coaxial cables. All of these standards and the cut-circle cavity are at a uniform temperature in the cryostat, and the switches are controlled by voltage pulses from a DC power supply (Hewlett-Packard E3610A) outside the cryostat. The network analyzer is an Agilent Technologies E8364C.

The cryostat includes two cylindrical vacuum chambers (aluminum) with one cylindrical thermal shield (copper) in between them. The switches, calibration standards, and the superconducting resonator are all contained in the inner vacuum chamber (designed temperature $4 \mathrm{~K}$, radius $16.5 \mathrm{~cm}$, and height $30.2 \mathrm{~cm}$ ). We use an Alcatel Drytel 31 Dry Vacuum Pump System to evacuate the inner and outer chambers to a pressure lower than $1 \times 10^{-6}$ atm and a Cryomech PT405 Pulse Tube Cryorefrigerator (with a water-cooled compressor) to cool down the resonator to the base temperature of $6.6 \mathrm{~K}$. The resonator is hung on the cold plate of the Cryorefrigerator, and we also use copper thermal straps to connect the cold plate and the resonator. The thermometer is attached in the lower part of the outside surface of the resonator. We also designed two copper clamps to mount and thermally anchor the cryogenic switches on the cold plate. The second layer of the cryostat is the cylindrical copper shield (designed temperature $40 \mathrm{~K}$, radius $17.8 \mathrm{~cm}$, and height $43.8 \mathrm{~cm})$. The third layer is the outer vacuum chamber (designed temperature $300 \mathrm{~K}$, radius $20.3 \mathrm{~cm}$, and height $55.9 \mathrm{~cm})$. For a thermal cycle, it takes about one day to pump the system to vacuum and cool down the resonator to thermal equilibrium at the base temperature, while warming-up takes about two days. The dwell time of the system at the base temperature can be longer than one week.

One advantage of using cryogenic switches is to save a great deal of time for the TRL calibration. One full thermal cycle of this cryostat takes about 3 days, so a multiple-thermal-cycle TRL calibration for 4 standards would require an additional 12 days for calibration. However, with the in-situ calibration system, we only need an additional 20 minutes for measuring the 4 standards within the same thermal cycle for measuring the superconducting cavity. The cryogenic switches also make the calibration process conveniently accessible each time the

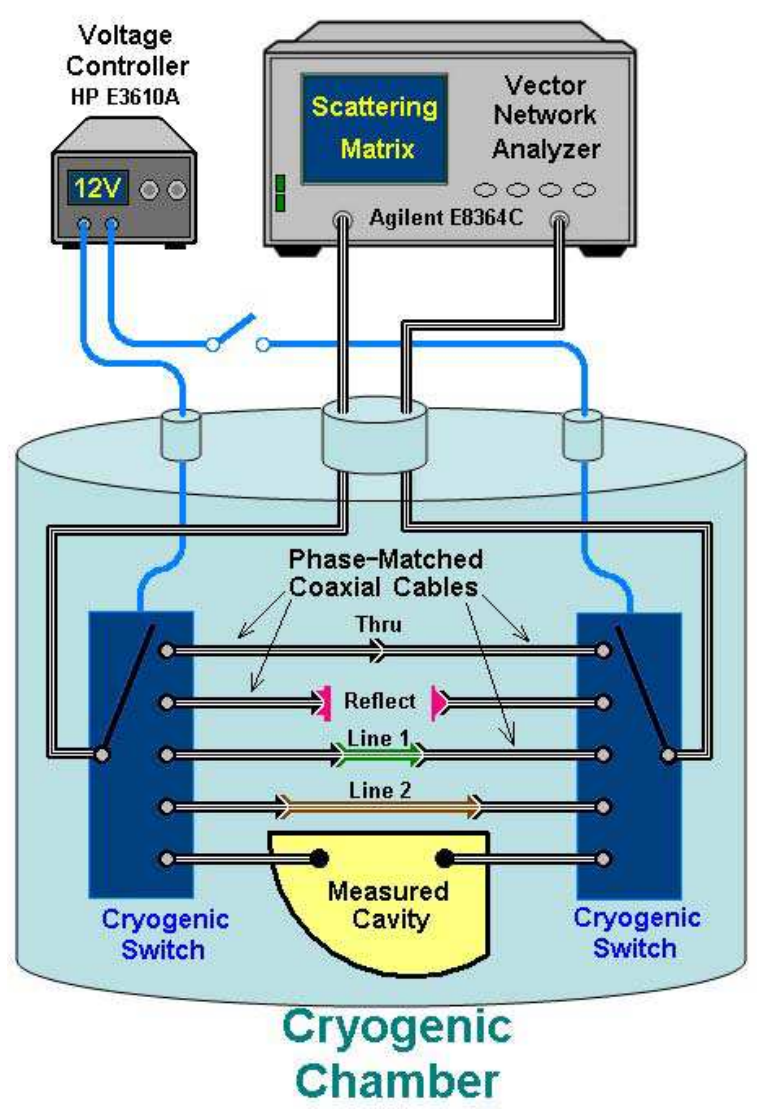

FIG. 2. Schematic experimental setup of the in-situ broadband cryogenic calibration system. The 5 pairs of phasematched coaxial cables have nearly identical length and are not shown to scale.

system environment is modified, for example, by changing the temperature, microwave power, or external magnetic field. We can adjust the equilibrium temperature of the cavity and standards by tuning the applied DC current to heating resistors installed in the cryostat. The applied microwave power can be controlled by the network analyzer. In addition to increasing the efficiency of the experiment, avoiding opening/closing the chamber for changing standards also reduces the uncertainties created by having different temperatures or different layouts of transmission lines in each measurement.

On the other hand, the disadvantage of using cryogenic switches is that the switches utilize 5 different pairs of transmission lines to connect to the standards or the cavity. The differences of the scattering matrices of these electrical paths are additional errors of the TRL calibration. We have measured the differences at room temperature, and the deviations from the switches are $|\triangle S|<0.01$; the deviations from the transmission lines are $|\triangle S|<0.06$. These errors can be reduced from the calibrated results by the pseudo-Open standard introduced in the following section.

The calibration system is also broadband because we install two Line standards. The TRL calibration is in- 
valid for frequencies where the phase difference between the Thru standard and the Line standard is too small (the phase difference should be greater than 20 degrees and less than 160 degrees) $\underline{16,19}$. To solve this problem, one can use two Line standards with different lengths and make sure that the problematic frequency bands do not overlap. In our experiment, one Line standard has problematic frequency bands near $7.7 \mathrm{GHz}$ and $15.3 \mathrm{GHz}$; the other Line standard has problematic frequency bands near $5.9 \mathrm{GHz}, 11.8 \mathrm{GHz}$, and $17.6 \mathrm{GHz}$. Therefore, we use the cryogenic switches to connect two different Line standards to achieve broadband calibration, for example, we can measure the scattering matrix continuously from 3 to $18 \mathrm{GHz}$.

We use MATLAB to operate the TRL calibration according to Rytting's algorithm $\stackrel{46}{ }$, and we combine the good frequency bands from the two Line standards to create a broadband result. We then use the pseudoOpen standard to remove the remaining errors in the TRL-calibrated data. One example of the result after these procedures is shown in Fig. 3 as the $\left|S_{11}\right|$ and $\left|S_{21}\right|$ of a single realization from 3 to $18 \mathrm{GHz}$. We can see the resonance density increase with frequency. In low frequency regions, the resonances are sharp and wellseparated, and $\left|S_{11}\right|$ is close to 1 in the frequencies away from the resonant frequencies. In high frequency regions, the resonance density increases, and the resonances start to overlap with each other. For example, we do not see the off-resonance background close to 1 in $\left|S_{11}\right|$ near 18 $\mathrm{GHz}$.

\section{Pseudo-Open Standard}

The scattering matrices calibrated by the TRL method still have many errors due to the fact that the practical standards and coaxial cables do not perfectly satisfy the assumptions of the TRL calibration. The remaining issues are: (1) the irreproducibility of the transmission lines and connectors in each electrical path, (2) the difference between the two reflectors in the Reflect standard, and (3) the impedance-mismatch and the imperfection of the Thru and Line standards. These errors are especially critical when $\left|S_{11}\right|$ and $\left|S_{22}\right|$ of the measured cavity are close to 1 . In our case, while the frequency is away from the resonant frequencies of the superconducting resonator, $\left|S_{11}\right|$ and $\left|S_{22}\right|$ are very close to 1 (i.e. the transmission coefficient $\left|S_{21}\right|$ is very close to 0 , and there is almost no absorption in the cavity) in the extremely low loss environment. Therefore, a small error can make $\left|S_{11}\right|$ or $\left|S_{22}\right|$ larger than 1 and cause non-physical results. This small error is also critical for analysis of the $2 \times 2$ impedance matrix $Z$, obtained by a bilinear transformation as $Z=Z_{0}\left(I_{2}+S\right) /\left(I_{2}-S\right)$ where $Z_{0}$ is the diagonal characteristic impedance matrix of the transmission lines $(50 \Omega)$ and $I_{2}$ is a $2 \times 2$ identity matrix. The denominator $\left(I_{2}-S\right)$ makes the impedance matrix sensitive to this small error when $S_{11}$ or $S_{22}$ are close to 1 .
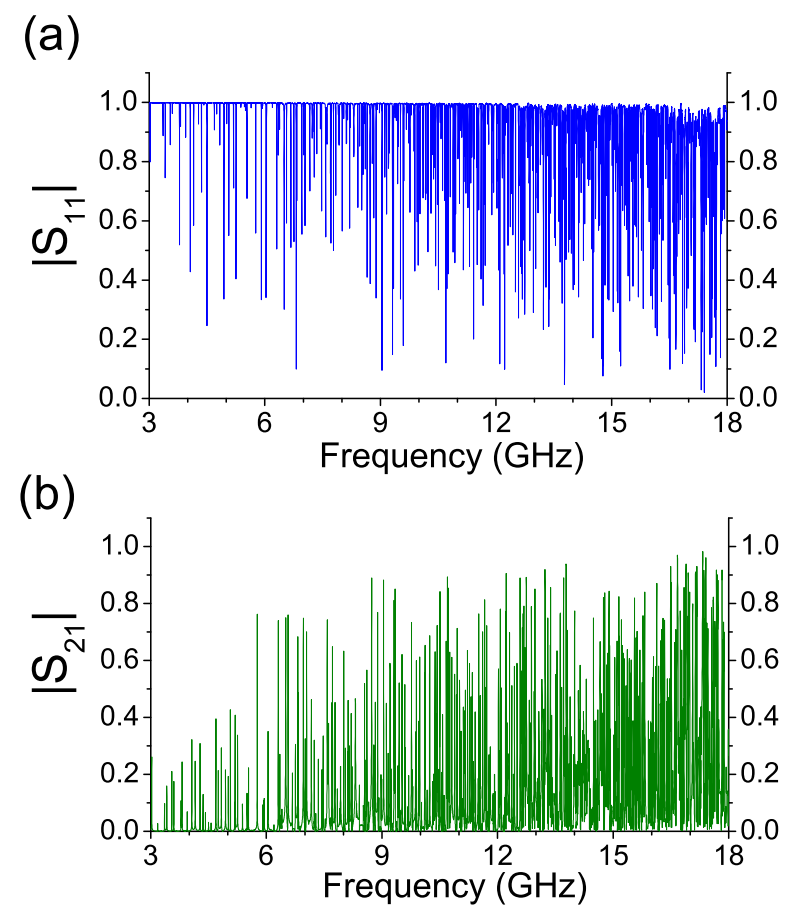

FIG. 3. The magnitude of (a) $\left|S_{11}\right|$ and (b) $\left|S_{21}\right|$ with highquality-factor resonances from 3 to $18 \mathrm{GHz}$ of a single realization of the superconducting cavity in the in-situ cryogenic calibration system. The data have been calibrated with the TRL calibration and corrected by the pseudo-Open method.

Figure 4 shows two examples of the raw data (without calibration) of the magnitude of the scattering matrix elements versus frequency. Because the loss parameter in the superconducting resonator is very low $(\alpha \ll 1$, i.e. high $Q)$, the curves of $\left|S_{\text {raw,11 }}\right|$ and $\left|S_{\text {raw,21 }}\right|$ show sharp and well-separated resonances on a smoothly varying background. Note that all degeneracies are broken in wave-chaotic billiards ${ }^{5}$. The background feature shows the influence of the transmission lines between the cavity and the network analyzer, and this is what we want to remove by calibration. With the cryogenic TRL calibration, we can eliminate most of the influence from the transmission lines. One example of the TRL-calibrated result $\left|S_{T R L, 11}\right|$ of a single realization is shown as the black curve in Fig. 5. As we expect for a superconducting resonator, now $\left|S_{T R L, 11}\right|$ is close to 1 at frequencies away from the resonant frequencies. However, there are still small variations in the background due to the systematic errors of the TRL calibration. Note that at some frequencies (e.g. near $15.64 \mathrm{GHz}$ ), the small error makes $\left|S_{T R L, 11}\right|>1$.

To solve this problem, we introduce the pseudo-Open standard by taking advantage of the multiple measurements of the 72 ensemble realizations of the cavity. In each realization, we only change the orientation of the Teflon perturber (see Fig. 1), and all of the other fea- 


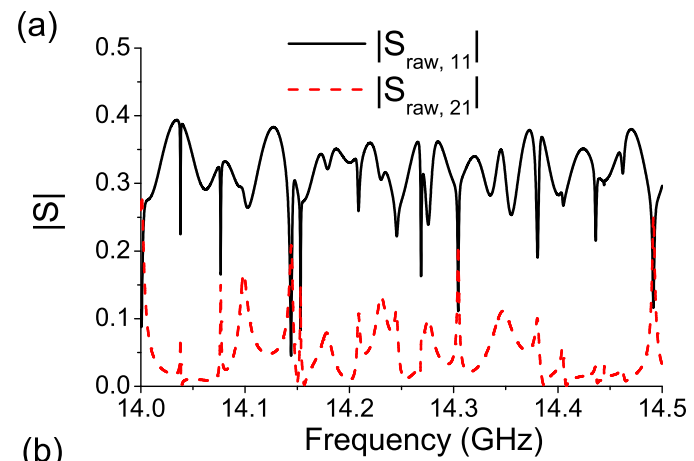

(b)

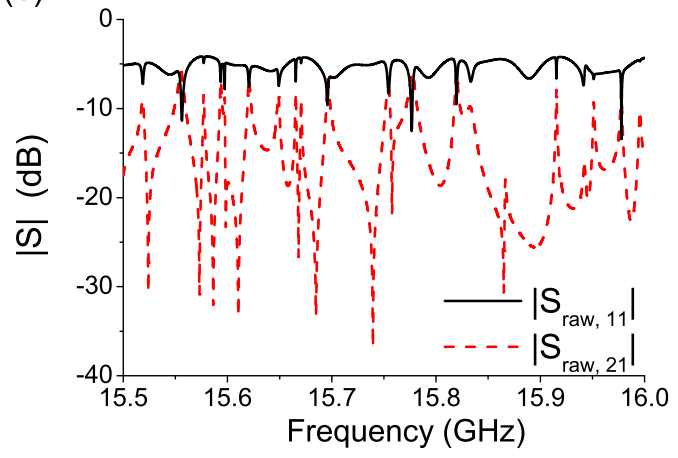

FIG. 4. The magnitude of raw measured scattering matrix element $S_{11}$ (black solid curves) and $S_{21}$ (red dash curves) versus frequency for the superconducting cut-circle microwave cavity at $6.6 \mathrm{~K}$. (a) An example with the frequency band from 14.0 to $14.5 \mathrm{GHz}$ plotted in linear scale; (b) another example with the frequency band from 15.5 to $16.0 \mathrm{GHz}$ plotted in semi-logarithmic scale.

tures, including the transmission lines, cavity volume, coupling, etc., remain the same. Therefore, by comparing the measured data of the 72 realizations, we see a systematically-varying background in the scattering matrices in all realizations, but the narrow and wellseparated resonances move to various frequencies. For illustration, Fig. 6 shows the quantity $\left(\left|S_{T R L, 11}\right|^{2}+\right.$ $\left.\left|S_{T R L, 21}\right|^{2}\right)^{1 / 2}$ in 12 realizations (each for a perturber orientation $30^{\circ}$ apart). Note that the resonances occur at varied frequencies in different realizations, but the off-resonance regions form a systematically-varying background.

We note that $U_{1} \equiv\left(\left|S_{T R L, 11}\right|^{2}+\left|S_{T R L, 21}\right|^{2}\right)^{1 / 2}$ and $U_{2} \equiv\left(\left|S_{T R L, 22}\right|^{2}+\left|S_{T R L, 12}\right|^{2}\right)^{1 / 2}$ are unity in a lossless system. For a very low loss system, RMT predicts that the $U_{1}$ and $U_{2}$ of an ensemble of realizations have statistical distributions $\frac{7}{}$ where most of the samples are close to 1 . If the cavity is weakly coupled through the ports to outside (i.e. $\left|S_{21}\right|$ and $\left|S_{12}\right|$ are closer to $0 ;\left|S_{11}\right|$ and $\left|S_{22}\right|$ are closer to 1), the distributions show that the samples of $U_{1}$ or $U_{2}$ are even closer to 1 . Therefore, if one takes the maximum of many realizations of $U_{1}$ or $U_{2}$ under these conditions, it should be very close to 1 .

We have constructed a numerical model to analyze the

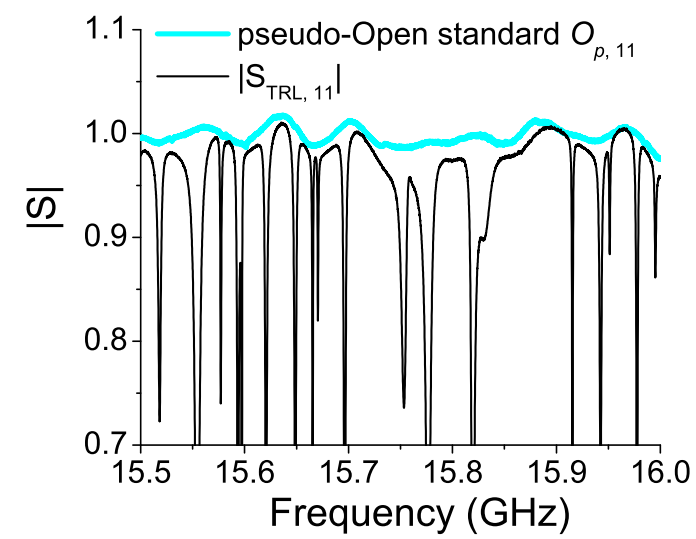

FIG. 5. The magnitude of TRL-calibrated $S_{11}$ (the black curve) and the pseudo-Open standard (the thicker light-blue curve) versus frequency.

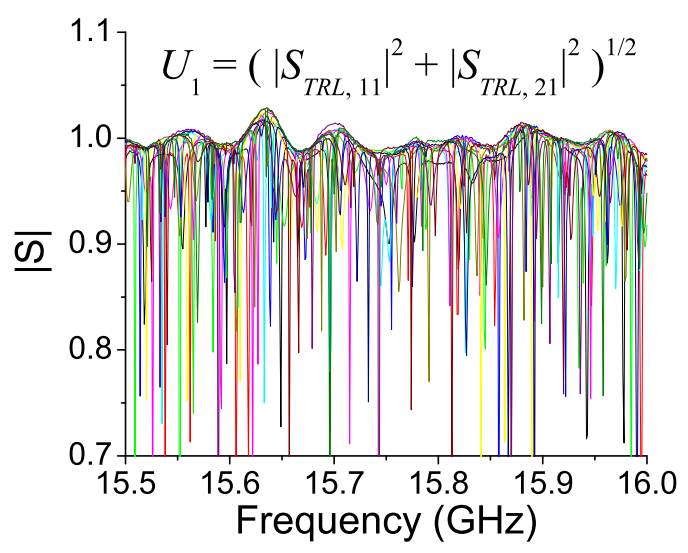

FIG. 6. Shown are 12 examples of different realizations of $U_{1}=\left(\left|S_{T R L, 11}\right|^{2}+\left|S_{T R L, 21}\right|^{2}\right)^{1 / 2}$ in varied colors versus frequency.

reason for the systematically-varying background. We use RMT and the RCM to numerically generate multiplerealization data to represent a superconducting cavity, and the maxima over the realization ensemble of $U_{1}$ and $U_{2}$ are very close to 1 for every frequency. However, if we combine these numerical cavity data with the measured data of our TRL standards and coaxial cables, and carrying out the same TRL calibration, we see a similar systematically-varying background. Therefore, this frequency-dependent feature represents a combination of all errors of the TRL calibration. According to our model, the major error in our experiment is from the Line standards, and it can cause errors for $S_{11}$ and $S_{22}$ of $|\triangle S|<0.1$ and an error for $S_{21}$ of $|\triangle S|<0.04$. The errors from the Thru standard and the Reflect standard are all smaller by a factor of 2 to 3 . 


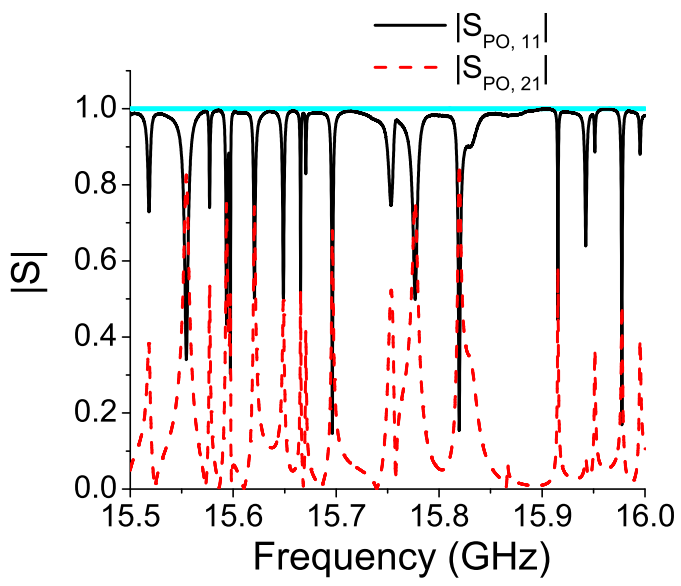

FIG. 7. The magnitude of TRL-calibrated and pseudo-Opencorrected $S_{11}$ (the black solid curve) and $S_{21}$ (the red dash curve) versus frequency. The horizontal blue line shows $|S|=$ 1.

In order to utilize this feature, we take the maximum values of $U_{1}$ and $U_{2}$ of the experimental data over the 72 realizations, and we define a frequency-dependent diagonal matrix $O_{p}$, where $O_{p, 11}$ (or $\left.O_{p, 22}\right)$ is the maximum of $U_{1}$ (or $U_{2}$ ) over the 72 realizations. In a very low loss system or a weakly coupled system, $O_{p}$ should be close to the identity matrix if the TRL calibration has no error. Thus, the frequency-dependent variations of $O_{p}$ represent the remaining errors in the experimental data after the TRL calibration. We call $O_{p}$ the pseudoOpen standard because $O_{p}$ is like an Open standard when we exclude all resonances (i.e. no energy is transmitted through the two ports). Figure 5 shows the pseudo-Open standard response $O_{p, 11}$ as the thicker light-blue curve. By utilizing the information from multiple measurements of the cavity in different realizations, the pseudo-Open standard helps to calibrate out the errors due to the deviations between the transmission lines connected to the TRL standards and the transmission lines connected to the cavity.

We simply remove the errors and obtain a TRLcalibrated and pseudo-Open-corrected scattering matrix $S_{P O}$ by

$$
S_{P O}=S_{T R L} O_{p}^{-1}
$$

Figure 7 shows the result of the pseudo-Open correction. The small systematic variations in the TRL-calibrated data $\left|S_{T R L, 11}\right|$ is removed after applying the pseudoOpen standard. Therefore, by using the TRL calibration with the pseudo-Open standard, we can have wellcalibrated data of the scattering matrix, and we are able to do further analysis of the statistics of the scattering matrices or the impedance matrices for wave chaos research 12 .

We also use our numerical model to test how the
pseudo-Open method resolves the errors from the TRL calibration. Since the pseudo-Open standard is based on the maximum values of $\left(\left|S_{T R L, 11}\right|^{2}+\left|S_{T R L, 21}\right|^{2}\right)^{1 / 2}$ and $\left(\left|S_{T R L, 22}\right|^{2}+\left|S_{T R L, 12}\right|^{2}\right)^{1 / 2}$, it contains no information about the phase of the elements of the scattering matrix. Therefore, it can only correct the magnitude of the scattering matrix, and the improvement is better when $\left|S_{11}\right|$ and $\left|S_{22}\right|$ are closer to 1 . Other limitations concern determination of the maxima of $\left(\left|S_{T R L, 11}\right|^{2}+\left|S_{T R L, 21}\right|^{2}\right)^{1 / 2}$ and $\left(\left|S_{T R L, 22}\right|^{2}+\left|S_{T R L, 12}\right|^{2}\right)^{1 / 2}$. In order to get a sampled maximum value close to the true maximum value, one needs to have a very low loss system or a weakly coupled system, or one needs to have a large number of realizations. Our experiment satisfies these requirements for the pseudo-Open standard, and according to the numerical test, the pseudo-Open method can remove the errors of the TRL calibration as measured by the statistical distributions of the magnitudes of the scattering matrix.

\section{Results and Test of RMT Predictions}

With the well-calibrated data of the scattering matrix, we can now apply the random coupling model $8,9,11,14$ to remove the system-specific features of the scattering matrix and reveal the universal statistics which are predicted by Random Matrix Theory ${ }^{7,12}$. Figure 8 shows a comparison of the universal statistics predicted by RMT (thicker light-blue curves) and the experimental data which are calibrated with cryogenic TRL calibration only (red dash curves) and also with pseudo-Open correction (black curves) in terms of the distributions of the RCMnormalized $\left|S_{11}\right|$ and $\left|S_{21}\right|$. The probability density functions (PDFs) of the experimental data are taken from all 72 realizations and in frequency from 14.0 to $16.0 \mathrm{GHz}$. The RMT predictions are the best-matched PDFs with a single parameter (the loss parameter $\alpha$ ), and $\alpha=0.02$ is the fit value. The results show that the pseudo-Open correction makes significant improvement in the PDFs when $\left|S_{11}\right|$ or $\left|S_{21}\right|$ are close to 1 . The non-physical features $(|S|>1$, seen in the TRL calibrated data in Fig. 5) are almost entirely eliminated with the correction of the pseudo-Open standard. The PDFs of the experimental data are not as smooth as the theoretical PDFs because we generate many more numerical samples to plot the theoretical curves. One can generalize these results to other resonant systems in which the modes can be perturbed by external means, such as strain, electric field, temperature, magnetic field, etc, to generate an ensemble of multiple measurements for the pseudo-Open method.

\section{CONCLUSION}

Superconducting microwave resonators are useful devices in many applications, and well-calibrated measurement of their scattering matrices is important. In this 


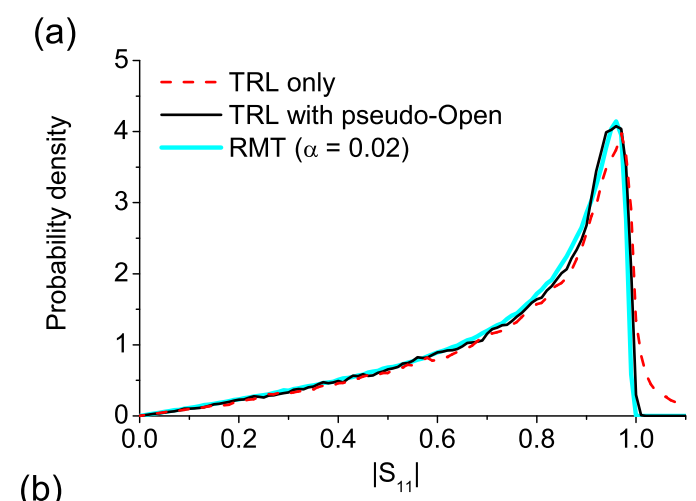

(b)

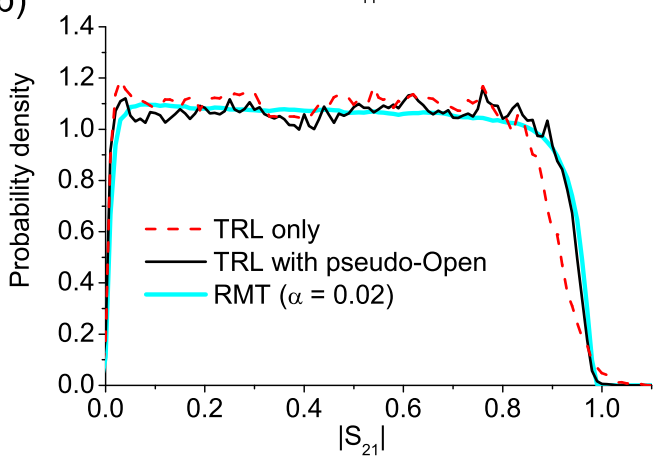

FIG. 8. The probability density of (a) $\left|S_{11}\right|$ and (b) $\left|S_{21}\right|$ of the RMT predictions (thicker light-blue curves), the experimental data from the superconducting cut-circle cavity at $6.6 \mathrm{~K}$ with TRL-calibration (red dash curves), and the data with TRLcalibration and pseudo-Open correction (black curves).

paper we demonstrate an in-situ broadband cryogenic calibration system where the calibration process is made dramatically more convenient by installing two cryogenic switches for single-thermal-cycle TRL calibration. We also introduce a pseudo-Open standard by taking advantage of the ensemble realizations of the superconducting cavity with a movable perturber and the feature of wellseparated resonances in an extremely low loss environment. Experimental data verify that the pseudo-Open standard can significantly improve the TRL-calibrated data. We show that the well-calibrated scattering matrices are beneficial for wave chaos research, and this work should broadly benefit various applications related to high-precision cryogenic measurement.

\section{ACKNOWLEDGMENTS}

We thank E. Ott and T. M. Antonsen for their helpful suggestions, the group of A. Richter (Technical University of Darmstadt) for graciously loaning the cut-circle billiard, and H. J. Paik and M. V. Moody for use of the pulsed tube refrigerator. We also acknowledge L. Ranzani for introducing us to the cryogenic switches. This work is funded by the ONR/Maryland AppEl Center
Task A2 (Contract No. N000140911190), the AFOSR under Grant No. FA95500710049, NSF-GOALI ECCS1158644, and the Center for Nanophysics and Advanced Materials (CNAM).

${ }^{1} \mathrm{H}$. Weinstock and M. Nisenoff, Microwave Superconductivity, (Kluwer Academic Publishers, Dordrecht, Netherlands, 1999).

${ }^{2}$ M. A. Hein, High-Temperature-Superconductor Thin Films at Microwave Frequencies, (Springer Tracts in Modern Physics 155, Springer, Heidelberg, Germany, 1999).

${ }^{3}$ H. Padamsee, J. Knobloch, and T. Hays, RF Superconductivity for Accelerators, (John Wiley \& Sons, New York, 1998).

${ }^{4}$ H.-D. Gräf, H. L. Harney, H. Lengeler, C. H. Lewenkopf, C. Rangacharyulu, A. Richter, P. Schardt, and H. A. Weidenmüller, Phys. Rev. Lett. 69, 1296 (1992); H. Alt, C. I. Barbosa, H.-D. Gräf, T. Guhr, H. L. Harney, R. Hofferbert, H. Rehfeld, and A. Richter, Phys. Rev. Lett. 81, 4847 (1998); C. Dembowski, B. Dietz, T. Friedrich, H.-D. Gräf, A. Heine, C. Mejía-Monasterio, M. Miski-Oglu, A. Richter, and T. H. Seligman, Phys. Rev. Lett. 93, 134102 (2004); A. Y. Abul-Magd, B. Dietz, T. Friedrich, and A. Richter, Phys. Rev. E 77, 046202 (2008); B. Dietz, T. Friedrich, H. L. Harney, M. Miski-Oglu, A. Richter, F. Schäfer, and H. A. Weidenmüller, Phys. Rev. E 81, 036205 (2010).

${ }^{5}$ H.-J. Stöckmann, Quantum Chaos, (Cambridge University Press, Cambridge, England, 1999).

${ }^{6}$ M. L. Mehta, Random Matrices, 2nd ed. (Academic Press, Boston, 1991); G. Akemann, J. Baik, and P. Di Francesco, The Oxford Handbook of Random Matrix Theory, (Oxford University Press, New York, 2011).

${ }^{7}$ P. W. Brouwer and C. W. J. Beenakker, Phys. Rev. B 55, 4695 (1997); C. W. J. Beenakker, Rev. Mod. Phys. 69, 731 (1997); Y. Alhassid, Rev. Mod. Phys. 72, 895 (2000).

${ }^{8}$ X. Zheng, T. M. Antonsen Jr., E. Ott, Electromagnetics 26, 3 (2006); Electromagnetics 26, 37 (2006).

${ }^{9}$ S. Hemmady, X. Zheng, E. Ott, T. M. Antonsen, and S. M. Anlage, Phys. Rev. Lett. 94, 014102 (2005); Phys. Rev. E 71, 056215 (2005); Phys. Rev. E 74, 036213 (2006); S. Hemmady, J. Hart, X. Zheng, T. M. Antonsen, E. Ott, S. M. Anlage, Phys. Rev. B 74, 195326 (2006).

${ }^{10}$ Y. V. Fyodorov, D. V. Savin, and H.-J. Sommers, J. Phys. A: Math. Gen. 38, 10731 (2005).

${ }^{11}$ J.-H. Yeh, J. Hart, E. Bradshaw, T. Antonsen, E. Ott, and S. M. Anlage, Phys. Rev. E 82, 041114 (2010).

12 J.-H. Yeh, T. M. Antonsen, E. Ott, and S. M. Anlage, Phys. Rev. E 85, 015202 (2012).

${ }^{13}$ M. Ławniczak, O. Hul, S. Bauch, P. Seba, and L. Sirko, Phys. Rev. E 77, 056210 (2008); M. Ławniczak, S. Bauch, O. Hul, and L. Sirko, Phys. Scr. T147, 014018 (2012).

${ }^{14}$ S. Hemmady, T. M. Antonsen, E. Ott, S. M. Anlage, IEEE Trans. Electromag. Compat. 54, 758 (2012).

${ }^{15}$ P. A. Mello, P. Pereyra, and T. H Seligman, Ann. Phys. 161, 254 (1985); J. Stein and H.-J. Stöckmann, Phys. Rev. Lett. 68, 2867 (1992); Y.-H. Kim, M. Barth, U. Kuhl, H.-J. Stöckmann, and J. P. Bird, Phys. Rev. B 68, 045315 (2003); U. Kuhl, M. Martínez-Mares, R. A. Méndez-Sánchez, and H.-J. Stöckmann, Phys. Rev. Lett. 94, 144101 (2005).

${ }^{16}$ http://na.tm.agilent.com/pna/help/latest/S3_Cals/TRL_Calibration.htm

17 J. L. Cano and E. Artal, Microwave Journal 52, 70 (2009); J. L. Cano de Diego, Ph.D. thesis, University of Cantabria, 2010.

${ }^{18}$ G. F. Engen and C. A. Hoer, IEEE Trans. Microw. Theory Tech. 27, 987 (1979).

${ }^{19}$ R. B. Marks, IEEE Trans. Microw. Theory Tech. 39, 1205 (1991).

${ }^{20} \mathrm{~A}$. Lewandowski and W. Wiatr, 74th ARFTG Microwave Measurement Symposium, Broomfield, Colorado, 2009 (DOI: 10.1109/ARFTG74.2009.5439110).

${ }^{21}$ J. C. Booth, D. H. Wu, and S. M. Anlage, Rev. Sci. Instrum. 65, 2082 (1994); J. C. Booth, D. H. Wu, S. B. Qadri, E. F. Skelton, M. S. Osofsky, A. Piqué, and S. M. Anlage, Phys. Rev. Lett. 77, 4438 (1996). 
${ }^{22}$ M. L. Stutzman, M. Lee, and R. F. Bradley, Rev. Sci. Instrum. 71, 4596 (2000).

${ }^{23}$ M. Scheffler and M. Dressel, Rev. Sci. Instrum. 76, 074702 (2005).

${ }^{24}$ K. Steinberg, M. Scheffler, and M. Dressel, Rev. Sci. Instrum. 83, 024704 (2012).

${ }^{25} \mathrm{~T}$. Reuss and J. Richard, IEEE Trans. Microw. Theory Tech. 48, 1286 (2000).

${ }^{26}$ H. Kitano, T. Ohashi, and A. Maeda, Rev. Sci. Instrum. 79, 074701 (2008).

${ }^{27}$ J. Laskar, J. J. Bautista, M. Nishimoto, M. Hamai, and R. Lai, IEEE Trans. Microw. Theory Tech. 44, 1178 (1996).

${ }^{28}$ J. C. Booth, J. A. Beall, D. C. DeGroot, D. A. Rudman, R. H. Ono, J. R. Miller, M. L. Chen, S. H. Hong, and Q. Y. Ma, IEEE Trans. Appl. Supercond. 7, 2780 (1997).

${ }^{29}$ K. T. Leong, J. C. Booth, and J. H. Claassen, J. Supercond. Nov. Magn. 19, 637 (2006).

${ }^{30}$ N. D. Orloff, J. Mateu, A. Lewandowski, E. Rocas, J. King, D. $\mathrm{Gu}, \mathrm{X}$. Lu, C. Collado, I. Takeuchi, and J. C. Booth, IEEE Trans. Microw. Theory Tech. 59, 188 (2011).

${ }^{31}$ V. Shemelin, M. Liepe, and H. Padamsee, NIM A 557, 268 (2006).

${ }^{32}$ M. S. Jun, S. W. Hwang, D. Y. Jeong, and D. Ahn, Rev. Sci. Instrum. 75, 2455 (2004)
${ }^{33}$ D. L. Birx and D. J. Scalapino, IEEE Trans. Magnetics MAG15, 33 (1979).

${ }^{34}$ L. Ranzani, L. Spietz, Z. Popovic, and J. Aumentado, IEEE Trans. Appl. Supercond. 22, 1500606 (2012).

${ }^{35}$ L. Spietz, K. Irwin, M. Lee, and J. Aumentado, Appl. Phys. Lett. 97, 142502 (2010).

${ }^{36}$ D. H. Slichter, Ph.D. thesis, University of California, Berkeley, 2011.

${ }^{37}$ http://web.physics.ucsb.edu/ martinisgroup/electronics.shtml. ${ }^{38}$ J. Juroshek, IEEE Trans. Microw. Theory Tech. 35, 457 (1987).

${ }^{39}$ T. E. MacKenzie and A. E. Sanderson, IEEE Trans. Microw. Theory Tech. 14, 29 (1966).

${ }^{40}$ J. P. Hoffmann, P. Leuchtmann, and R. Vahldieck, Proc. European Microwave Conference, 388 (2007).

${ }^{41}$ G. Vandersteen, Y. Rolain, J. Schoukens, and A. Verschueren, IEEE Trans. Microw. Theory Tech. 45, 1027 (1997).

${ }^{42}$ S. Ree and L. E. Reichl, Phys. Rev. E 60, 1607 (1999).

${ }^{43}$ B. Dietz, A. Heine, A. Richter, O. Bohigas, and P. Leboeuf, Phys. Rev. E 73, 035201(R) (2006).

${ }^{44}$ B. Dietz, T. Friedrich, H. L. Harney, M. Miski-Oglu, A. Richter, F. Schäfer, and H. A. Weidenmüller, Phys. Rev. E 78, 055204 (2008).

${ }^{45}$ A. Richter, Phys. Scr. T90, 212 (2001).

${ }^{46}$ D. Rytting, "Network analyzer error models and calibration methods", Proc. ARFTG/NIST Short Course RF Meas. Wireless World, 2001. 